\title{
Wind Fragility for Sign Structure in Korea with Chemical Anchor Connection
}

\author{
Viriyavudh $\mathrm{Sim}^{1}$, SeongDo Kim ${ }^{1}$, WooYoung Jung ${ }^{1}$ \\ ${ }^{1}$ Gangneung-Wonju National University, Department of Civil Engineering, 25457 Gangneung, Republic of Korea
}

\begin{abstract}
Damage to domestic facilities by strong winds and typhoon is on the rise in recent year. Typical sign structure used in Korea is found on the edge of concrete building, which could be easily affected by strong winds. Therefore, this study focused on sign structure among various vulnerable facilities. The evaluation of wind fragility for sign structure was carried out considering the failure of anchor at their connection to the concrete building. Moreover, pull-out and shear tests were performed to determine the resistance capacity of anchor used in this study. Monte Carlo Simulation method was used to generate random wind loads on sign structure. Additionally, fragility parameters were determined based on the failure criteria of connection anchor. Results show that diameter of the installed anchor was the dominant factor affecting the performance of sign structure subjected to strong wind.
\end{abstract}

\section{Introduction}

Per Korea Typhoon White Book from Korea National Typhoon Center 2011 [1], typhoon disaster in Korea in recent year has been on the rise. The risk due to typhoon causes devastating loss on economy and human life. Therefore, the risk assessment for structure subject to strong wind loads is required. In fact, probabilistic risk assessment (PRA) has emerged as an increasingly popular analysis tool, especially in the last decade. For instance, Lee and Rosowsky [2] and Ellingwood, Rosowsky, Li and Kim [3] analyse fragility curve for wood frame structure subjected to lateral wind loads by means of Monte Carlo Simulation (MCS) method, which is a part of analytical fragility development. The method was based on statistical wind load parameters determined by Ellingwood and Tekie [4] by means of Delphi questionnaire with expert of this field. Moreover, by combining the statistical parameters with nominal wind load parameters in standard design code from American Society of Civil Engineering (ASCE 7-10) [5], it is applicable to variety of structure types.

PRA is a systematic and comprehensive methodology to evaluate risks associated with every lifecycle aspect of structural and non-structural component. Recent disasters around the world have highlighted the necessity of risk assessment for all types of structure, especially those located in high density region or metropolitan area, which prone to cause higher economic losses and social disruption. Advertisement sign structures in South Korea are those mostly found in crowded region and mostly constructed in the form of attachment to the side or on the roof of concrete structure. Most published studies use regression technique with post-disaster investigations or historical recorded data to develop fragility curves [6]. However, this approach cannot be used to develop fragility curves for structure in South Korea because of the limitation of post-disaster and historical data [7].

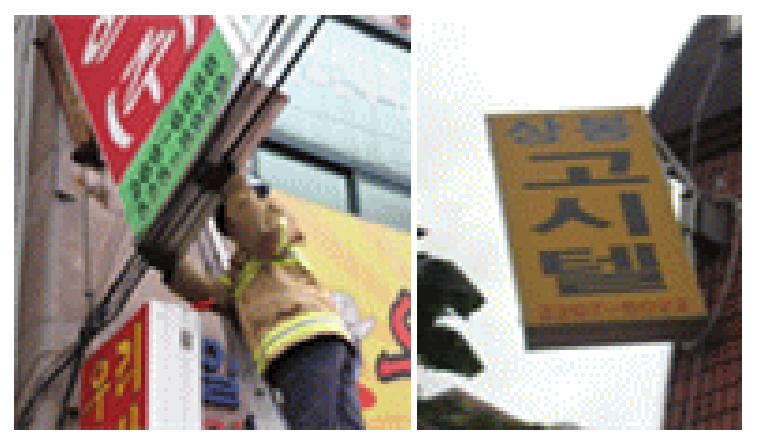

Fig. 1. Failure of sign structure.

In this paper, a probabilistic approach was presented to predict the wind-induced damage of sign structure. This approach used Monte Carlo Simulation method to generate damage information for structural component. Additionally, considering the experts' opinions of related companies, the damage of sign structure frequently occurs at the attachment surface, which can be seen in Figure 1; thus, the connection anchors were selected as vulnerable member in this study. Subsequently, the simulation compared probabilistic wind loads and resistance strength of structural component based on experimental test data of chemical anchor frequently used in South Korea. Finally, the wind fragility of the chemical anchor was evaluated. Multiple types of chemical anchor were considered based on their length and diameter. 


\section{Performance evaluation of chemical anchor}

Pull-out and shear test was performed to determine resistance capacity of chemical anchor. The experiment was performed with four types of anchor based on their dimension. The dimensions of the anchors were selected from the most commonly used in the market with lengths of $50 \mathrm{~mm}$ and $100 \mathrm{~mm}$ and diameters of $10 \mathrm{~mm}$ and 12 $\mathrm{mm}$. Chemical anchor was shown in Figure 2. The experiment was carried out according to: penetration depth and anchor diameter.

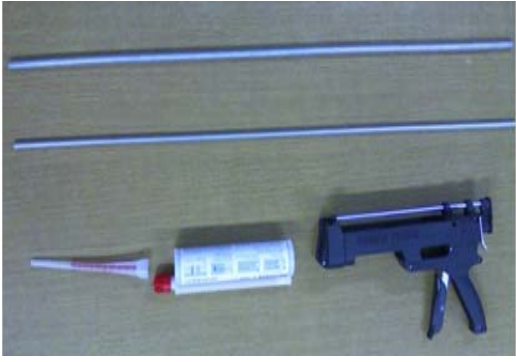

Fig. 2. Chemical anchor.

The performance of the chemical anchor was measured three times per experimental variable and the mean value was obtained for each experimental parameter. The preparation of concrete block and experiment procedure was shown in Figure 3. In Figure 3(a)-(c), the concrete bock was constructed and anchor hole was drilled and clean up for installation of anchor. Then, the chemical anchor in Figure 2 was installed. Finally, the pull-out and shear tests were performed as shown in Figure 3(d).

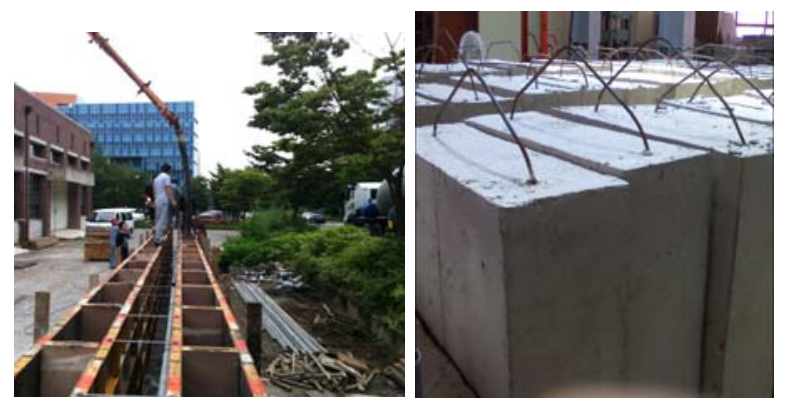

(a). Construction of concrete specimen

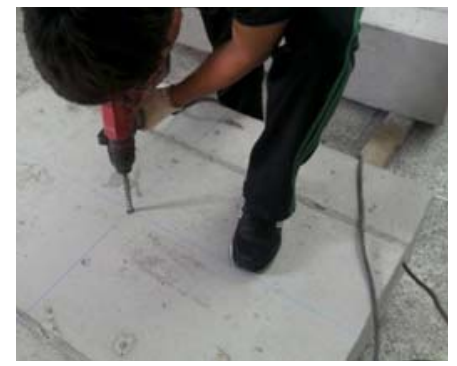

(b). Hole drilling for anchor installation

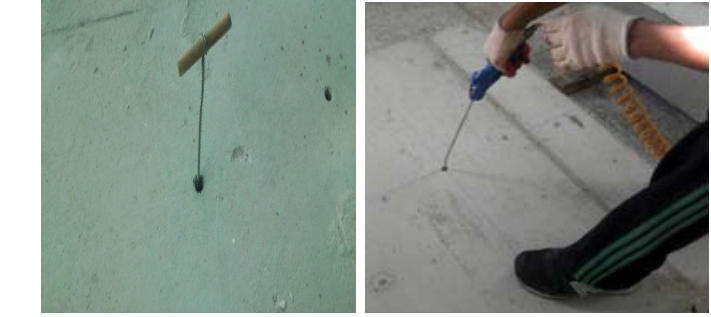

(c). Clean up for anchor installation

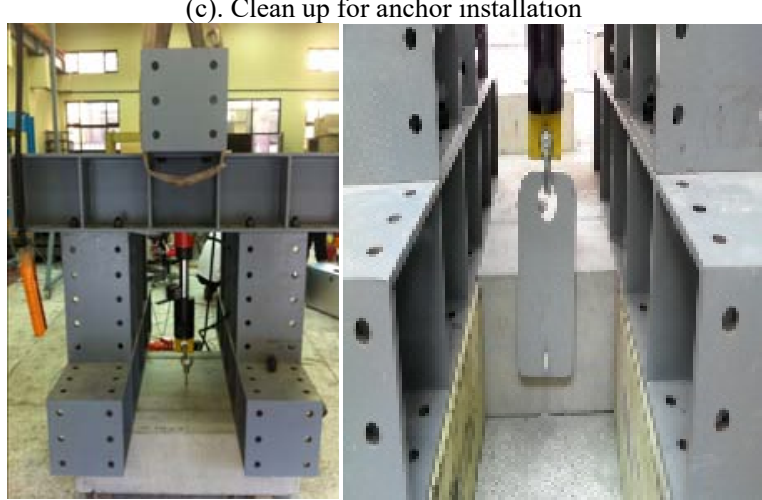

(d). Pull-out and shear test

Fig. 3. Experimental procedure.

Results of the pull-out and shear tests were shown in Figure 4 and Figure 5, respectively. In Figure 4, (a) the failure due to anchor pull-out and (b) the forcedisplacement curves for three tests were shown. Likewise, in Figure 5, the failure form and forcedisplacement curves were shown for shear test. Additionally, Table 1 and Table 2 show the results of displacements and forces for all four types of anchor selected for this study.

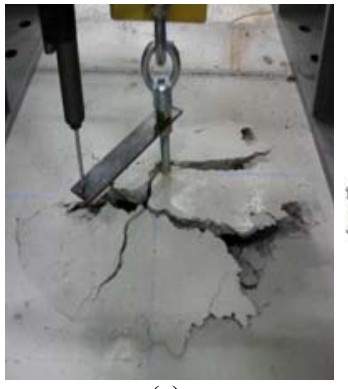

(a) (b)

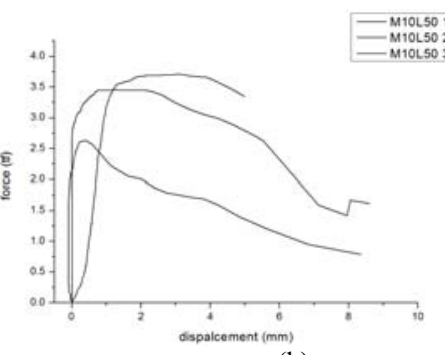

Fig. 4. Pull-out test results.

Table 1. Chemical anchor pull-out test results.

\begin{tabular}{|c|c|c|c|}
\hline No. & Anchor & $\begin{array}{c}\text { Displacement } \\
(\mathrm{mm})\end{array}$ & $\begin{array}{c}\text { P } \\
(\text { ton })\end{array}$ \\
\hline 1 & M10L50 & 3.852 & 3.367 \\
\hline 2 & M10L100 & 3.473 & 3.907 \\
\hline 3 & M12L50 & 4.514 & 4.623 \\
\hline 4 & M12L100 & 4.627 & 9.247 \\
\hline
\end{tabular}




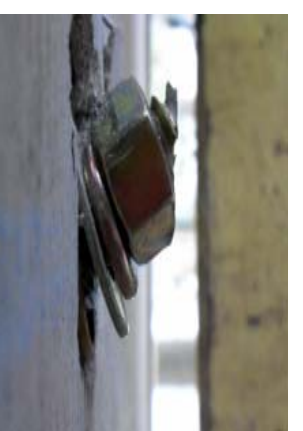

(a)

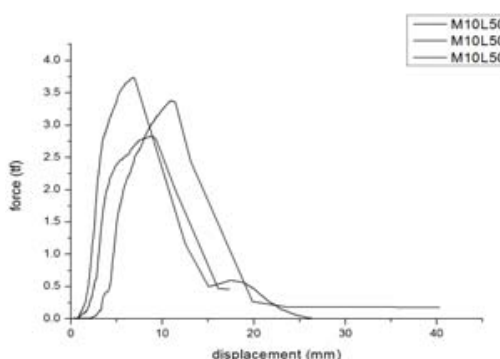

(b)
Fig. 5. Shear test results.

Table 2. Chemical anchor shear test results.

\begin{tabular}{|c|c|c|c|}
\hline No. & Anchor & $\begin{array}{c}\text { Displacement } \\
(\mathrm{mm})\end{array}$ & $\begin{array}{c}\text { P } \\
\text { (ton) }\end{array}$ \\
\hline 1 & M10L50 & 10.995 & 3.317 \\
\hline 2 & M10L100 & 16.528 & 3.657 \\
\hline 3 & M12L50 & 8.631 & 5.421 \\
\hline 4 & M12L100 & 11.105 & 5.977 \\
\hline
\end{tabular}

In both experiments, the strength of anchor bolts was measured using a hydraulic double acting cylinder (TDC-3030) and a load-controllable server, and the displacement values were also measured by linear variable differential transformer (LVDT). The resistance capacity was estimated based on the experimental results. Finally, we derived and evaluated the wind fragility based on the performance of these anchors.

\section{Structure model setting}

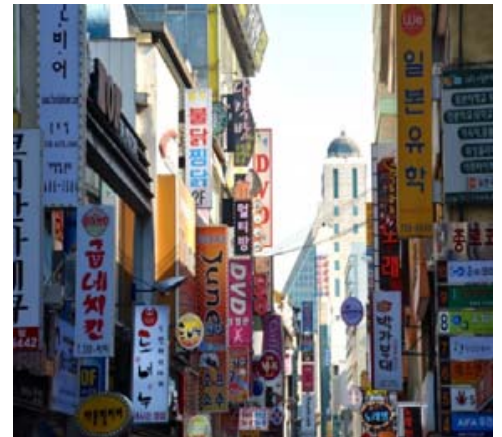

(a)

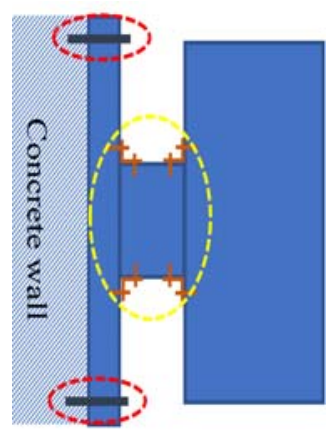

(b)
Fig. 6. Typical sign structure in Korea.

In South Korea, advertisement is commonly placed on the sign structure connected to the side of concrete building in the crowded area as can be seen in Figure 6(a). More specifically, the sign structure model in this study was set up as shown in Figure 6(b) for wind fragility evaluation. The advertisement panel's dimension was $0.8 \mathrm{~m} \times 3.2 \mathrm{~m}$. In this diagram, the panel was connected to the steel plate through the rigid connection box with 8 screws and the steel plate connected to concrete wall with 4 anchors. Failure modes of the sign structure due to strong wind include connection angle dropout and connection anchor failure; however, in this study, the connection box was assumed to be perfectly rigid, only the anchor failure was considered.

\section{Evaluation of sign structure wind fragility}

\subsection{Wind loads}

ASCE 7 (2010) defines two types of structural elements subjected to wind load: (1) main wind-force resisting systems (MWFRS), and (2) components and cladding $(\mathrm{C} \& \mathrm{C})$. The wind load acting on solid freestanding sign structures shall be determined with provision for MWFR in ASCE 7 (2010) with the following:

$$
F=q_{h} G C_{f} A_{s}
$$

where $q_{h}=$ velocity pressure evaluated at height $\mathrm{h}$ (top edge of sign panel), $G=$ gust-effect factor, $C_{f}=$ net force coefficient, and $A_{s}=$ gross area of the solid freestanding sign in $\left(\mathrm{m}^{2}\right)$. The velocity pressure evaluated at height $z$ in ASCE 7 (2010) is given by:

$$
q_{z}=0.613 K_{z} K_{z t} K_{d} V^{2}
$$

where $q_{h}$ is equivalent to $q_{z}$ at height $h, K_{z}=$ velocity pressure exposure factor, $K_{z t}=$ topographic factor, $K_{d}=$ wind directionality factor, and $V=$ basic wind speed in $(\mathrm{m} / \mathrm{s})(3$-second gust wind speed at $10 \mathrm{~m}$ and in open terrain).

Summary of statistical wind load parameters used in this study was shown in Table 3. Normal distribution of parameters $K_{z}, K_{d}$, and $G$ were obtained from previous Delphi study of wind parameters by [2] and [4].

Table 3. Summary of statistical wind load parameters.

\begin{tabular}{|c|c|c|c|c|}
\hline \multirow{2}{*}{ Parameter } & Category & Mean & SD & CDF \\
\hline \multirow{2}{*}{$K_{z}$} & Exposure B & 0.89 & 0.17 & Normal \\
\cline { 2 - 5 } & Exposure C & 1.08 & 0.15 & Normal \\
\cline { 2 - 5 } & Exposure D & 1.28 & 0.18 & Normal \\
\hline \multirow{2}{*}{$K_{d}$} & MWFRS & 0.89 & 0.14 & Normal \\
\hline \multirow{3}{*}{$G$} & Exposure B & 0.77 & 0.09 & Normal \\
\cline { 2 - 5 } & Exposure C & 0.83 & 0.10 & Normal \\
\cline { 2 - 5 } & Exposure D & 0.83 & 0.07 & Normal \\
\hline
\end{tabular}




\begin{tabular}{|c|c|}
\hline$C_{f}$ & Deterministic (1.93) \\
\hline$K_{z t}$ & Deterministic (1.00) \\
\hline
\end{tabular}

\subsection{Probability of failure of anchor bolt}

Failure of sign structure due to wind loads occurs when force generated by wind exceeds the resistance capacity of connection anchor. Thus, the failure criteria for anchor bolts can be written in term of the basic (random) variables as:

$$
g(x)=R-F
$$

where $R=$ resistance capacity of the anchor bolt, $F=$ wind loads acting on sign panel that apply tension and shear loading to anchor bolts. Failure of anchor bolts can be defined as a condition where $g(x)<0$. The wind loads, and hence the probability, is a function of the basic wind speed $(V)$ squared, see (1) and (2).

Monte Carlo Simulation method was used to generate random wind loads $(F)$ based on their statistical distribution in Table 3. For each wind speed, MCS generated random velocity exposure factor, wind directionality factor, and force coefficients by sampling from their normal distribution function parameters. Subsequently, by comparing wind loads demand with anchor resistance capacity, the failure of anchor could be determined. These comparisons were repeated 10,000 times to determine the probability of failure at each wind speed. Furthermore, by repeating this procedure for the next increment of wind speed, the probability of failure for anchor could be determined for all wind speeds.

\subsection{Wind fragility modelling of anchor bolt}

The fragility parameters of a structural system commonly modelled using a lognormal cumulative distribution function as the following [8]:

$$
\operatorname{Fr}(V)=\Phi\left[\frac{\ln (x)-\mu_{R}}{\sigma_{R}}\right]
$$

in which $\Phi(\cdot)$ = standard normal cumulative distribution function, $\mu_{R}=$ logarithmic median of capacity $R$ (in units that are dimensionally consistent with demand), and $\sigma_{R}=$ logarithmic standard deviation of capacity $R$.

\section{Results and discussions}

Wind fragility parameters $\mu$ and $\sigma$ were determined by maximum likelihood estimation (MLE) method [9]. Figure 7-9 show the wind fragility of chemical anchor installed in sign structure in region with wind exposure category B. Anchor No. 4 (M12L100) showed the highest resistance to wind loads, where their median wind speed had the highest value, i.e. $103 \mathrm{~m} / \mathrm{s}$. Then, it was followed by anchor No. 3 (M12L50), No. 2 (M10L100) and No. 1 (M10L50) with the median wind speed $93 \mathrm{~m} / \mathrm{s}, 78 \mathrm{~m} / \mathrm{s}$ and $74 \mathrm{~m} / \mathrm{s}$, respectively.
Accordingly, there was a minor difference between median wind speed of anchor with the same diameter, i.e. $10 \mathrm{~m} / \mathrm{s}$ for $12 \mathrm{~mm}$ anchor and $4 \mathrm{~m} / \mathrm{s}$ for $10 \mathrm{~mm}$ anchor. However, for anchor with the same length, a more significant difference between their median wind speed was shown. For anchors with $50 \mathrm{~mm}$ length, the difference between their median wind speed were $19 \mathrm{~m} / \mathrm{s}$; anchor with $100 \mathrm{~mm}$ length shown a difference of $25 \mathrm{~m} / \mathrm{s}$.

This trend was also shown in the other two wind exposure categories. Therefore, it could be concluded that the difference of probability of failure for anchor with the same diameter $(10 \mathrm{~mm}$ and $12 \mathrm{~mm})$ was less than the difference between anchor with the same length $(50 \mathrm{~mm}$ and $100 \mathrm{~mm})$. Thus, the diameter of chemical anchor installed for the connection of sign structure and concrete wall can significantly affect the performance of sign structure system.

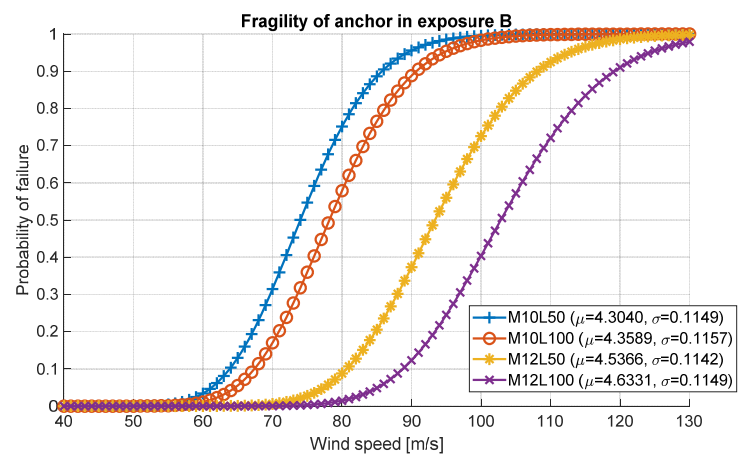

Fig. 7. Wind fragility of chemical anchor in sign structure in wind exposure B.

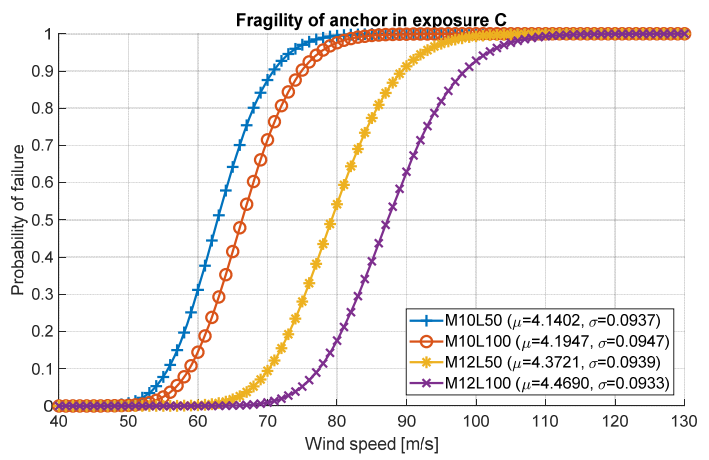

Fig. 8. Wind fragility of chemical anchor in sign structure in wind exposure $\mathrm{C}$.

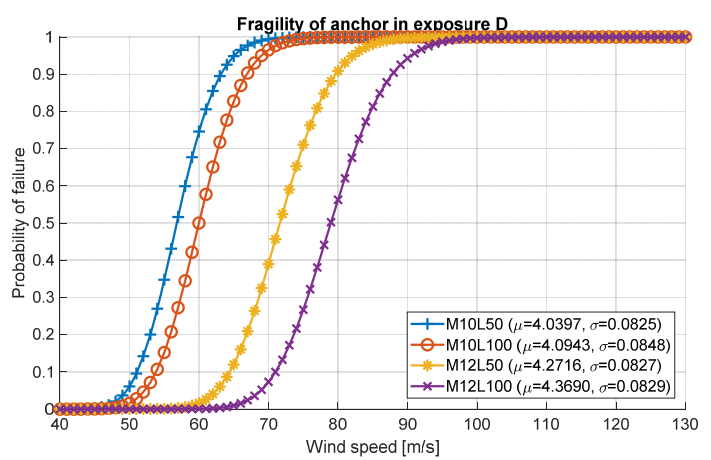

Fig. 9. Wind fragility of chemical anchor in sign structure in wind exposure D. 


\section{Summary and conclusions}

Performance of advertisement sign structure was evaluated based on the performance of their connection anchor to the concrete wall. Probability of failure for sign structure were determined analytically with Monte Carlo Simulation method. Moreover, fragility parameters were fitted to lognormal cumulative distribution function by using the maximum likelihood estimation method. Results were shown for anchor installed in sign structure in region with wind exposure $\mathrm{B}, \mathrm{C}$ and $\mathrm{D}$. Additionally, the difference between median wind speed of each anchor had also been discussed.

The results illustrated that anchor No. 4 (M12L100) shown the highest resistance; while, anchor No. 1 (M10L50) had the lowest resistance capacity. The differences between median wind speed of each anchor shown the significant effect of anchor diameter on the performance of sign structure exposed to strong wind. It could be concluded that the diameter of chemical anchor was more significant than their length in application where the chemical anchor was used in connection between sign structure and concrete wall.

In conclusion, usage of this methodology could lead to a more predictable structure performance and could facilitate the introduction of performance-base design guidelines for sign structure in high wind regions. Fragilities such as those presented here also can convolve with wind hazard curves to develop a risk assessment tool, which can evaluate the potential impact of a natural hazard in public planning and mitigate the consequent economic losses and social disruption.

\section{Acknowledgment}

This research was supported by a grant [MOIS-DP2015-05] through the Disaster and Safety Management Institute funded by Ministry of the Interior and Safety of Korean government.

\section{References}

1. National Typhoon Center, Typhoon White Book 2011, Publication Registration Number: 111360016-000001-01, (2011)

2. K. H. Lee, D. V. Rosowsky, Fragility curves for woodframe structures subjected to lateral wind loads, Wind Struct., 9, 217-230 (2006)

3. B. R. Ellingwood, D. V. Rosowsky, Y. Li, J. H. Kim, Fragility assessment of light-frame wood construction subjected to wind and earthquake hazards, J. Struct. E, 130, 1921-1930 (2004)

4. B. R. Ellingwood, and P. B. Tekie, Wind load statistics for probability-based structural design, J. Struct. E, 125, 453-463 (1999)

5. American Society of Civil Engineers, Minimum design loads for buildings and other structures, ASCE, 7 (2010)

6. A. D. Cope, Predicting the vulnerability of typical residential buildings to hurricane damage, Doctoral dis., University of Florida (2004)

7. H. Ham, S. Lee, H. Kim, Development of typhoon fragility for industrial buildings, Proc. 7th AsiaPacific Conf. Wind E, Taipei (2009)

8. K. Porter, Beginner's guide to fragility, vulnerability, and risk, Ency. Eq. E, 235-260 (2015)

9. J. W. Baker, Efficient analytical fragility function fitting using dynamic structural analysis, Eq. Spect., 31, 579-599 (2015) 